\title{
Fuling-Guizhi Herb Pair in Coronary Heart Disease: Integrating Network Pharmacology and In Vivo Pharmacological Evaluation
}

\author{
Bailu Duan $\mathbb{D}^{1},{ }^{1}$ Lintao Han $\mathbb{D}^{2},{ }^{2}$ Shuping Ming, ${ }^{3,4}$ JingJing Li $\mathbb{D},{ }^{1}$ Qiong Wang $\mathbb{D},{ }^{1}$ \\ Dongning Zhang, ${ }^{2}$ Guangyu Tian $\left(\mathbb{0},{ }^{1}\right.$ and Fang Huang $\mathbb{\circledR}^{1}$ \\ ${ }^{1}$ College of Basic Medicine, Hubei University of Chinese Medicine, Wuhan 430065, China \\ ${ }^{2}$ Pharmacy School, Hubei University of Chinese Medicine, Wuhan 430065, China \\ ${ }^{3}$ First Clinical College, Hubei University of Chinese Medicine, Wuhan 430065, China \\ ${ }^{4}$ Department of Neurology, Hubei Provincial Traditional Chinese Medical Hospital, Wuhan 430065, China
}

Correspondence should be addressed to Guangyu Tian; tgy_ccp@stmail.hbtcm.edu.cn and Fang Huang; hf_ccp@hbtcm.edu.cn

Received 31 January 2020; Revised 14 April 2020; Accepted 28 April 2020; Published 18 May 2020

Academic Editor: Luigi Milella

Copyright (c) 2020 Bailu Duan et al. This is an open access article distributed under the Creative Commons Attribution License, which permits unrestricted use, distribution, and reproduction in any medium, provided the original work is properly cited.

The Fuling (Poria cocos)-Guizhi (Cinnamomi ramulus) herb pair (FGHP) is a commonly used traditional Chinese herbal formula with coronary heart disease (CHD) treatment potential. However, the mechanism of FGHP in the treatment of CHD was still unclear. In this study, the action targets and underlying mechanism of FGHP against CHD were successfully achieved by combined network pharmacology prediction with experimental verification. 76 common targets were screened out by overlapping the chemical-protein data of FGHP and CHD-related targets. Then, two key targets were further selected for verification by using western blot analysis after analyzing PPI, GO function, and KEGG pathway. Results indicated FGHP could alleviate CHD syndromes and regulate inflammatory responses in acute myocardial ischemia rats, and the reduction of expression of TNF- $\alpha$ and IL-6 in myocardial tissue would be one of its possible underlying mechanisms. Our work demonstrated that network pharmacology combined with experimental verification provides a credible method to elucidate the pharmacological mechanism of FGHP against CHD.

\section{Introduction}

Coronary heart disease $(\mathrm{CHD})$ is a common type of heart disease and one of the most common causes of human deaths in the world $[1,2]$. The World Health Organization (WHO) estimates that approximately 17.9 million people die of cardiovascular diseases in the world every year [3] and 7.4 million die of CHD [4]. It is well-known that CHD is an archetypical multifactorial disease [5]. Single-target drugs usually fail as a cure for this multifactorial disease [6]. In addition, repeated or continuous high-dose of a single specific drug can produce drug resistance $[6,7]$. Therefore, the discovery of new multitarget drugs is highly necessary.

Traditional Chinese medicine (TCM), mainly Chinese herbal medicine, has been used for thousands of years to treat $\mathrm{CHD}$ and its related diseases, as it is characterized by multiple components, and it can treat diseases through multiple pathways and targets $[8,9]$. Herb pairs (combination of two herbs) are the simplest form of herbal combination and compatibility [10], which have therapeutic features and clinical significance in Chinese herbal medicine. The Fuling-Guizhi herb pair (FGHP) is a famous formula originated from Linggui Zhugan Decoction, a well-known Chinese medicinal formula consisting of four Chinese herbal medicines-Poria cocos (Chinese name, Fuling), Cinnamomi ramulus (Chinese name, Guizhi), Atractylodes macrocephala Koidz. (Chinese name, Baizhu), and Licorice (Chinese name, Gancao), recorded in the classic ancient book "JinGuiYaoNue" written by Zhongjing Zhang. FGHP has been commonly used clinically for the treatment of CHD. However, the mechanism by which FGHP helps to treat CHD remains unclear, which impedes its further clinical application and spread in some degree. 
Network pharmacology recently has become a new and a powerful approach to systematically reveal the principles and function of a complex biological system along with the rapid progress of bioinformatics $[11,12]$, and it has become an efficient way to preliminarily observe action at the system level and to explore the potential pharmacological mechanism of TCM at the molecular level $[12,13]$.

Therefore, network pharmacology-based study was adopted in this study to investigate underlying action mechanism of FGHP in CHD. First, the potential action of FGHP was predicted using a network pharmacological method. Subsequently, an isoproterenol- (ISO-) induced acute myocardial ischemia (AMI) experiment was carried out on rats to confirm the network analysis-based data.

\section{Materials and Methods}

\subsection{Network Pharmacology-Based Analysis}

2.1.1. Active Component Screening. Firstly, chemical compounds in Fuling (FL) and Guizhi (GZ) were obtained from the Encyclopedia of Traditional Chinese Medicine (ETCM, http://www.nrc.ac.cn: 9090/ETCM), the Traditional Chinese Medicine Integrated Database (TCMID, http://www. megabionet.org/tcmid/), and the Traditional Chinese Medicine Systems Pharmacology Database (TCMSP, http:// tcmspw.com/tcmsp.php). The active components were the filtered by combining oral bioavailability (OB) $\geq 30 \%$ and drug-likeness (DL) index $\geq 0.18$ as suggested by the TCMSP database.

\subsubsection{Target Prediction of FGHP against CHD. SWISS} (http://www.swisstargetprediction.ch/) and STITCH (http:// stitch.embl.de/) databases were used to identify the potential human protein targets of FGHP's active compounds. At the same time, CHD-related human genes were downloaded from OMIM (https://omim.org/) and DisGeNET (https:// www.disgenet.org/) databases. Then, the candidate targets of FGHP against CHD were obtained by overlapping the above targets with a Venn diagram (http://bioinfogp.cnb.csic.es/ tools/venny/index.html).

2.1.3. Gene Ontology and Pathway Enrichment Analysis. The gene ontology (GO) and Kyoto Encyclopedia of Genes and Genomes (KEGG) enrichment analysis of the candidate targets were carried out using the DAVID (https://david. ncifcrf.gov/) to obtain the related functions and pathways.

2.1.4. Protein-Protein Interaction (PPI) Data. The proteinprotein interaction (PPI) data were gathered from STRING (https://string-db.org/) database, which supplies information regarding the predicted and verified experimental interactions of proteins [14], with species simply limited to "Homo sapiens" and interaction score $>0.7$ (high confidence). The top 10 proteins with higher levels of correlation were collected as the center targets for FGHP against CHD.
2.1.5. Network Construction. Based on these results, a compound-target-pathway network composed of compounds, corresponding targets, and their related potential pathways was constructed and displayed using Cytoscape 3.7.1 (http://cytoscape.org/), which is a software package for visualizing network analysis [15].

\subsection{Evaluation of Anti-CHD Properties of FGHP In Vivo}

2.2.1. Animals and Drugs. Male SD rats weighing 220-250 g (6-8 weeks) were obtained from the Hubei Experiment Animal Research Center (Wuhan, China). All rats were housed in the SPF laboratory animal room, Hubei University of Chinese Medicine (Wuhan, China), and maintained in suitable conditions with a $12 \mathrm{~h}$ light/dark cycle and room temperature at $22 \pm 2^{\circ} \mathrm{C}$ and $50 \pm 10 \%$ humidity. They had free access to food and water. The study protocol was approved by Animal Ethics Committee of Hubei University of Chinese Medicine (HUCMS-201903001). Fuling and Guizhi were purchased from TCM pharmacy of Wuhan Hospital of Traditional Chinese Medicine (Wuhan, China). The FGHP was obtained by mixing the two herbs (Fuling and Guizhi) at a mass ratio of $1: 1$ and then grinding them into powder. Propranolol tablets were purchased from Ya Bang Pharmaceutical Factory (Changzhou, China). Isoproterenol (ISO) hydrochloride was purchased from Southwest Pharmaceutical Co., Ltd. (Chongqing, China).

2.2.2. Experimental Process. Rats were randomly divided into the control group and three administration groups ( $n=6$ per group): ISO, ISO + FGHP $(1.8 \mathrm{~g} / \mathrm{kg})$, and ISO+ Propranolol (Pro, $10 \mathrm{mg} / \mathrm{kg}$ ). The rats in the two ISO+ groups were pretreated by oral gavage administration with propranolol or FGHP, while the rats in the control group and the ISO group were pretreated by oral gavage administration with equal volumes of $0.9 \%$ saline once a day for 2 weeks. Afterwards, the rats in all three administration groups were subcutaneously injected with ISO $(85 \mathrm{mg} / \mathrm{kg})$ for two consecutive days $[16,17]$. FGHP dosage for the rats was calculated in proportion to human doses $(20 \mathrm{~g} /$ day per adult) using body surface area conversion.

At the end of the experiment, electrocardiograms (ECGs) were recorded under pentobarbital anesthesia using a BL-420F biological data acquisition and analysis system. Blood was collected via the abdominal aorta. The serum was separated by centrifugation at $4^{\circ} \mathrm{C}$ and 3,000 rpm for $15 \mathrm{~min}$ and subsequently stored at $-20^{\circ} \mathrm{C}$ for biochemical analysis. The heart tissues were dissected and immediately frozen in liquid nitrogen or fixed in $10 \%$ paraformaldehyde for western blot or histological analysis.

2.2.3. Biochemical Analysis. The serum creatine kinase-MB (CK-MB) and lactate dehydrogenase ( $\mathrm{LDH})$ levels were measured by using commercial kits and following the product instructions (CUSABIO BIOTECH Co., Ltd., Wuhan, China). 
2.2.4. Histopathological Analysis. The myocardial tissues were dissected and then fixed in $10 \%$ paraformaldehyde overnight followed by processing of dehydrating, paraffin embedding, sectioning, and staining (H\&E). Morphological structure of each specimen was examined and photographed by using a light microscope (BH-2, Olympus, Japan).

2.2.5. Western Blot Analysis. The total protein from the myocardial tissues were extracted as described previously [17]. Next, the protein was separated on $12 \%$ SDS-PAGE and electrophoretically transferred onto PVDF membrane. After blocking with $5 \%$ nonfat milk in TBS- $0.1 \%$ Tween-20 (TBST) for 1 hour, the membranes were incubated with the following primary antibodies: anti-IL-6 (\#12912), anti-TNF$\alpha$ (\#6954) (CST, Massachusetts, USA), and anti- $\beta$-actin (BOSTER, Wuhan, China) at $1: 1000$ dilution at $4^{\circ} \mathrm{C}$ overnight. Following washing in TBST, the membranes were incubated with corresponding horseradish peroxidaseconjugated secondary antibodies. Then, the protein bands were visualized with a BeyoECL Plus (P0018M, Beyotime, Shanghai, China). Densitometry analysis of each band was performed using the BioRad Quantity One software. $\beta$-Actin protein was used as the internal control for semiquantitative analysis.

2.3. Statistical Analysis. Data were expressed as the mean\pm SEM. GraphPad Prism 5 software (San Diego, CA, United States) was used for the statistical analysis and graphics. Unpaired $t$-test was used to analyze statistical comparisons between two groups. Multiple comparisons were assessed by one-way ANOVA. $P<0.05$ was assumed as statistically significant.

\section{Results}

\subsection{Results of Network Pharmacology-Based Analysis}

3.1.1. Active Components and Target Identification of FGHP. Totally, 33 components of Fuling (FL) and 220 components of Guizhi (GZ) were obtained from the ETCM, TCMID, and TCMSP (Table S1). All active components should satisfy the filtering rules, OB index $\geq 30 \%$ and DL value $\geq 0.18$. After screening, a total of 18 compounds of FGHP were collected, including 11 in FL and 7 in GZ (as shown in Table 1). By fishing for targets, 277 potential targets were found for FGHP. Detailed information of the targets is provided in Table S2.

3.1.2. Targets of FGHP against CHD. A total of 912 CHDrelated targets were obtained from the DisGeNET and OMIM. The detailed information of the targets is shown in Table S3. Then, the predictive targets of FGHP were overlapped with the CHD-related targets. Totally, 76 candidate targets of FGHP against CHD were identified, excluding any duplicate targets (Figure 1). These targets are listed in Table S4.
3.1.3. GO and Pathway Enrichment Analysis. To explore the multiple mechanisms of FGHP on CHD from a systematic level, gene ontology (GO) analysis and KEGG pathway enrichment of the 76 candidate targets were performed. In total, 96 enriched GO terms and 48 pathways were identified $(P<0.05$, Table S5). Top $5 \mathrm{GO}$ functional categories in biological process, cellular component, and molecular function and 15 remarkable pathways were selected and are presented in Figures 2(a) and 2(b).

3.1.4. PPI Network of Target Genes. The STRING tool was employed to obtain PPI relationships for the 76 target genes. With a confidence score of $>0.7$ was selected, the network of PPI relationships has 76 nodes and 237 edges (Figure 3(a)). The top 10 proteins with higher levels of connectivity were collected as the center targets for FGHP against CHD (Figure 3(b)).

The center target genes, which might play an important role in CHD progression, were peroxisome proliferator activated receptor gamma (PPARG), matrix metalloproteinase 9 (MMP9), mitogen-activated protein kinase 8(MAPK8), mitogen-activated protein kinase 3(MAPK3), interleukin 6 (IL6), epidermal growth factor receptor (EGFR), vascular endothelial growth factor A (VEGFA), tumor necrosis factor (TNF), retinoid $\mathrm{X}$ receptor alpha (RXRA), and prostaglandin-endoperoxide synthase 2 (PTGS2).

3.1.5. Compound-Target-Pathway Network. Based on the all above information, we constructed a compound-targetpathway network (Figure 4) to explain the mechanism of FGHP in treating CHD. This network had 43 nodes (18 compounds, 10 targets, and 15 pathways) and 102 edges, in which yellow squares, green circles, and red circles correspond to active components, their corresponding target proteins, and potential pathways involved, respectively.

\subsection{Results of Pharmacodynamic Study of FGHP}

3.2.1. The Effect of FGHP on ECG. As shown in Figure 5, the control group showed normal ECG readings (Figure 5(a)), whereas rats injected with $85 \mathrm{mg} / \mathrm{kg}$ ISO showed a marked increase in the ST segment, when compared with the normal controls. These results suggest that the acute myocardial ischemia (AMI) model was successfully established. Though FGHP and propranolol pretreatments could not completely inhibit the ISO-induced elevation of the ST segment, obvious decreases in the ST segment were observed in FGHP and Pro pretreated groups compared with the ISO group (Figure 5(b)).

3.2.2. Effects of FGHP on Serum Cardiac Markers. The serum concentrations of creatine kinase-MB (CK-MB) and lactic dehydrogenase (LDH) in the ISO group were significantly increased than those in the control group $(P<0.01)$. Pretreatment with FGHP and propranolol markedly inhibited the elevation in the levels of serum CK-MB and 
TABLE 1: A list of the active compounds in FGHP.

\begin{tabular}{|c|c|c|c|c|}
\hline No. & Compound & $\begin{array}{l}\mathrm{OB} \\
(\%)\end{array}$ & $\mathrm{DL}$ & Herb \\
\hline 1 & $\begin{array}{l}\text { (2R)-2-[(3S, 5R, 10S, 13R, 14R, 16R, 17R)-3,16-Dihydroxy-4,4,10,13,14-pentamethyl-2,3,5,6,12,15,16,17- } \\
\text { octahydro-1H-cyclopenta[a]phenanthren-17-yl]-6-methylhept-5-enoic acid }\end{array}$ & 30.93 & 0.81 & $\mathrm{FL}$ \\
\hline 2 & Trametenolic acid & 38.71 & 0.8 & FL \\
\hline 3 & Cerevisterol & 37.96 & 0.77 & FL \\
\hline 4 & Ergosterol peroxide & 40.36 & 0.81 & FL \\
\hline 5 & $3 \beta$-Hydroxy-24-methylene-8-lanostene-21-oic acid & 38.7 & 0.81 & FL \\
\hline 6 & Pachymic acid & 33.63 & 0.81 & FL \\
\hline 7 & Poricoic acid A & 30.61 & 0.76 & FL \\
\hline 8 & Poricoic acid B & 30.52 & 0.75 & FL \\
\hline 9 & Poricoic acid C & 38.15 & 0.75 & FL \\
\hline 10 & Hederagenin & 36.91 & 0.75 & FL \\
\hline 11 & Dehydroeburicoic acid & 44.17 & 0.83 & FL \\
\hline 12 & $(-)$-Taxifolin & 60.51 & 0.27 & GZ \\
\hline 13 & $\beta$-Sitosterol & 36.91 & 0.75 & GZ \\
\hline 14 & Sitosterol & 36.91 & 0.75 & GZ \\
\hline 15 & $(+)$-Catechin & 54.83 & 0.24 & GZ \\
\hline 16 & ent-Epicatechin & 48.96 & 0.24 & GZ \\
\hline 17 & Taxifolin & 57.84 & 0.27 & GZ \\
\hline 18 & Peroxyergosterol & 44.39 & 0.82 & $\mathrm{GZ}$ \\
\hline
\end{tabular}

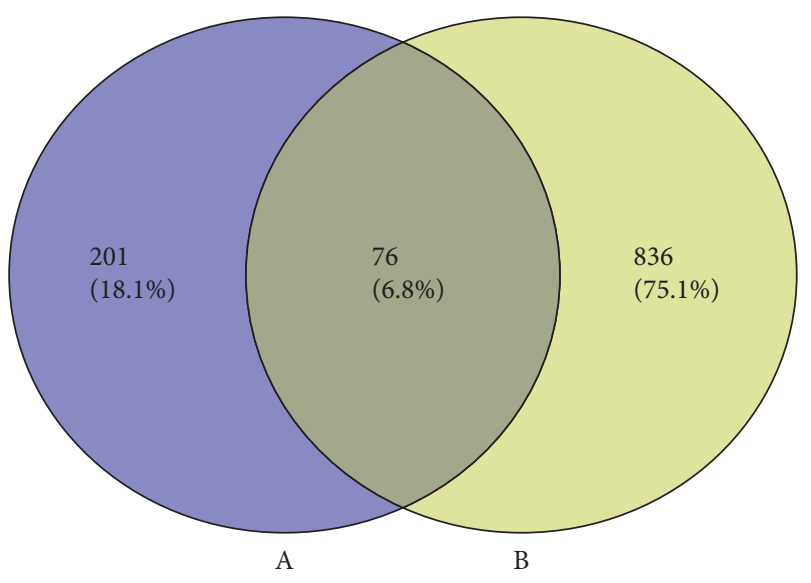

Figure 1: Overlapping targets between FGHP (A) and CHD (B).

LDH of the ISO group $(P<0.01)$ as shown in Figures 6(a) and $6(\mathrm{~b})$.

\subsubsection{The Effect of FGHP on Histopathological Features.} Compared to the control group (Figure 7(a)), the ISO group rats (Figure $7(\mathrm{~b})$ ) showed more frequently pathological changes, such as myocardial cell swelling, separation of cardiac muscle fibers, interstitial edema, and inflammatory cell infiltration. Such histological changes were significantly alleviated in the propranolol (Figure 7(c)) and FGHP (Figure $7(\mathrm{~d})$ ) pretreatment groups compared to the ISO group.

\subsection{Experimental Validation of the Network Pharmacology.} Based on the network pharmacology analysis, to verify the results derived using the PPI and compound-target-pathway network, we chose the high two related targets (TNF- $\alpha$ and IL-6) for experimental validation. As shown in Figure 8, the protein levels of TNF- $\alpha$ and IL- 6 were significantly increased in the ISO group compared with those in the control group $(P<0.01)$. Pretreatment with FGHP or propranolol showed that TNF- $\alpha$ and IL-6 expression decreased obviously in the ISO + FGHP or the ISO + Pro groups compared with the ISO group $(P<0.05)$.

\section{Discussion}

CHD is a complex disease and the final manifestation of various pathological insults [5, 18]. Developing novel comprehension multicomponent synergy strategies are vital for conquering complex and multifactorial diseases [19]. It is believed multicomponent and multitarget drugs can effectively reduce side effects and improve adaptive resistance, thus improving the possibility of overcoming diseases $[7,19]$. It is generally known that for over 5,000 years, traditional Chinese medicine (TCM) derived from numerous herbal formulas or herb pairs to form multiingredient herbal medicine was used to cure diseases [20, 21]. However, it is actually recognized that the intricacy of ingredients, multiple targets, pathways, and mechanisms still restrains the development of TCMs.

The advent of network pharmacology provides a novel opportunity to explore possible pharmacological mechanisms of TCMs [22]. At present, a lot of studies have made an effort to adopt network pharmacology to investigate the intricacy of ingredients, targets, pathways, and mechanisms of action of herb pairs or herbal formulas [23-25]. Thus, we used the network pharmacology method to explore the possible pharmacological mechanisms of FGHP related to CHD in the present study.

Network pharmacological analysis of FGHP showed that 18 compounds and 76 target genes were associated to CHD. According to the results of pathway enrichment, we believe that effects of FGHP against CHD may be due to the fact that 


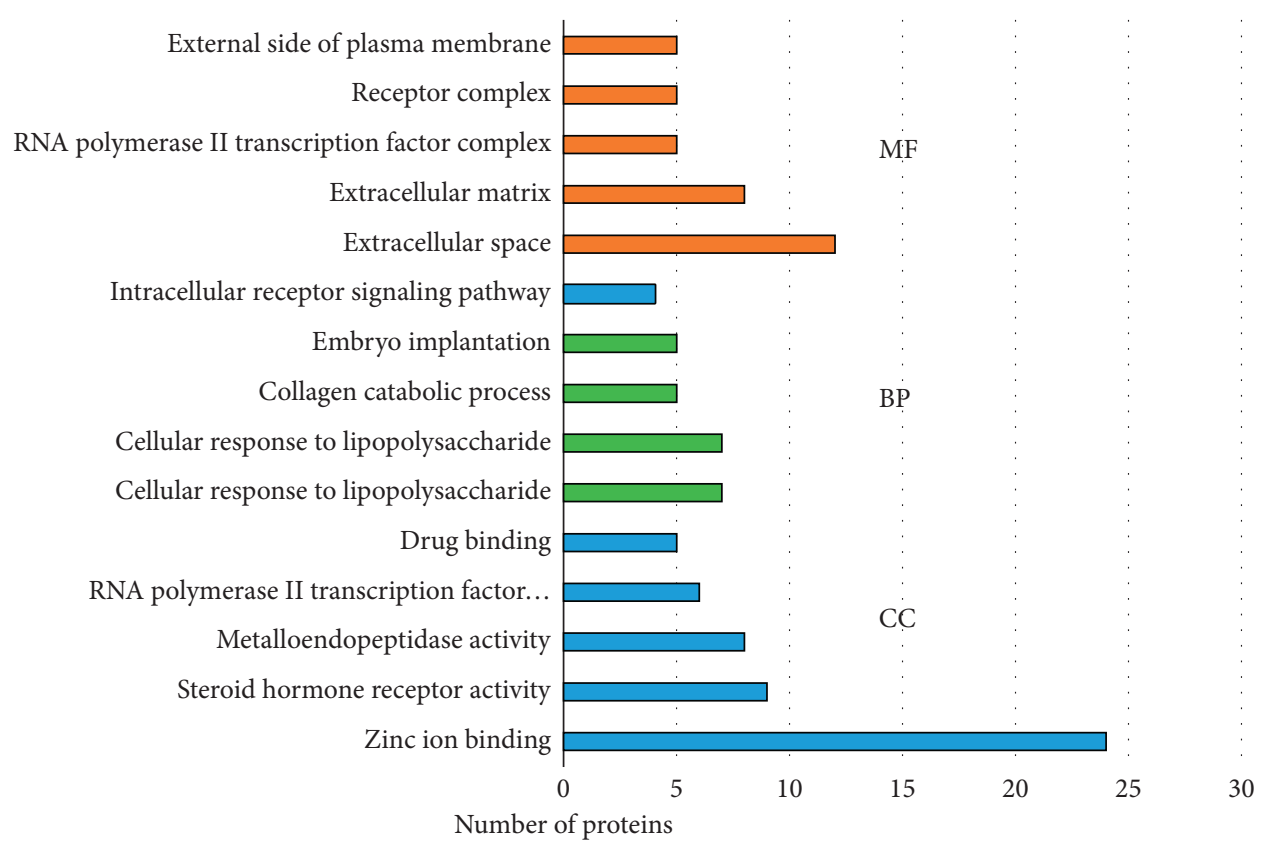

(a)

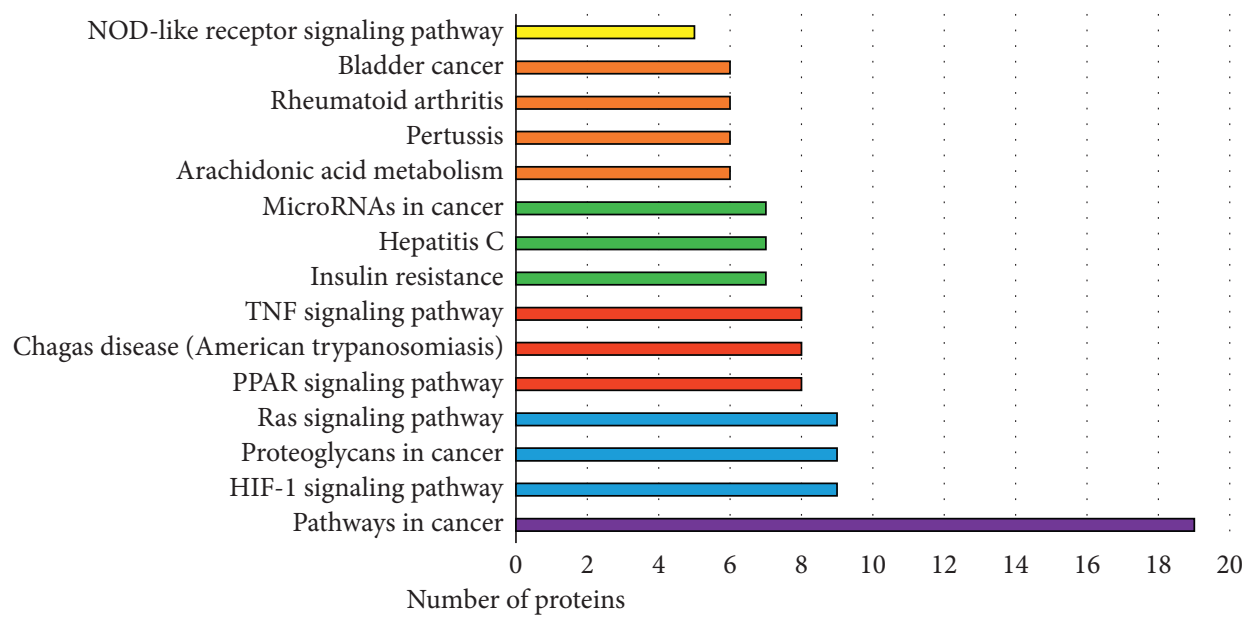

(b)

Figure 2: GO (a) and pathway (b) enrichment analysis by DAVID.

FGHP can simultaneously target multiple pathways like pathways in cancer, HIF-1 signaling pathway, PPAR signaling pathway, TNF signaling pathway, and so on (shown in Figure 2(b)). Based on the results of PPI analysis, PPARG, MMP9, MAPK8, MAPK3, IL6, EGFR, VEGFA, TNF, RXRA, and PTGS2 were elected as key targets (shown in Figure 3(b)). It was proposed that the occurrence and development of CHD is widely considered as a chronic inflammatory process characterized by highly specific cytokine response $[26,27]$. TNF- $\alpha$ is a key inflammatory mediator and is considered to be a major indicator of the instability of plaques during the formation of thrombi [28]. IL-6, a circulating cytokine known for its regulation of inflammatory reaction, plays a vital role in atherogenesis and thrombosis [29]. In fact, many studies have shown that various cytokines such as tumor TNF- $\alpha$ and IL- 6 are involved in the pathogenesis of CHD [30-32].
FGHP originated from Linggui Zhugan Decoction, which has actually been medically used for lots of years in the treatment of several diseases, including cardiovascular diseases and metabolic diseases [33]. It was reported that Linggui Zhugan Decoction suppressed production of inflammatory mediators (IL6, TNF- $\alpha$, and IL-1 $\beta$ ) in lipopolysaccharide- (LPS-) induced cardiomyocyte injury model rats [34]. Linggui Zhugan Decoction significantly inhibited the elevation in the levels of serum IL6, IL18, and IL-10 of the chronic heart failure rats [35]. More importantly, some active ingredients in FGHP such as hederagenin and beta-sitosterol were reported to act on TNF- $\alpha$ and IL- 6 to exert their anti-inflammatory properties $[36,37]$. So, TNF- $\alpha$ and IL6 were selected as the evaluation indicators to observe the therapeutic effect and possible mechanism of FGHP in ISO-induced AMI rats. 


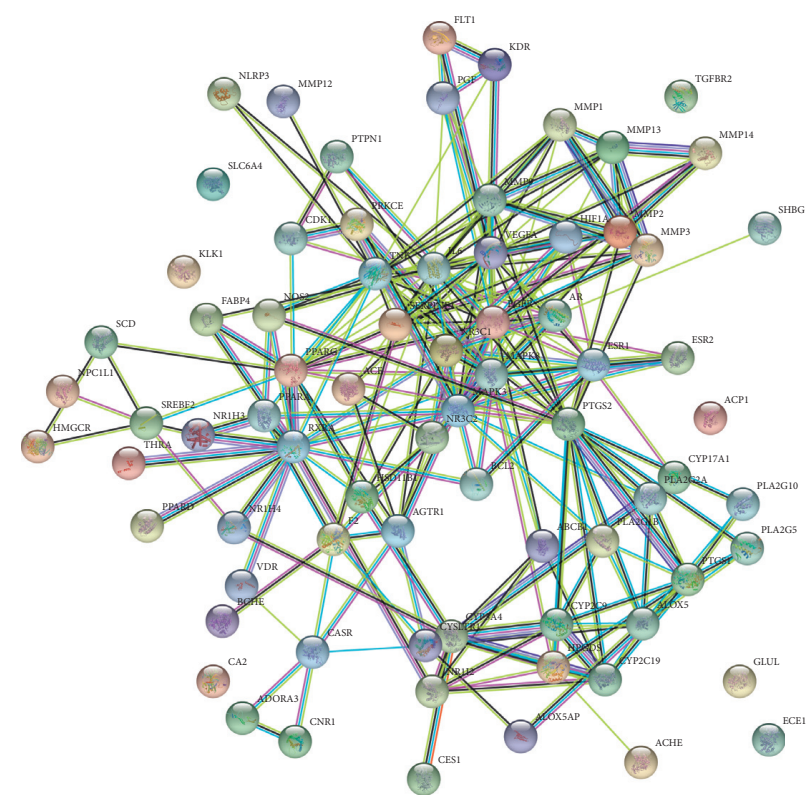

(a)

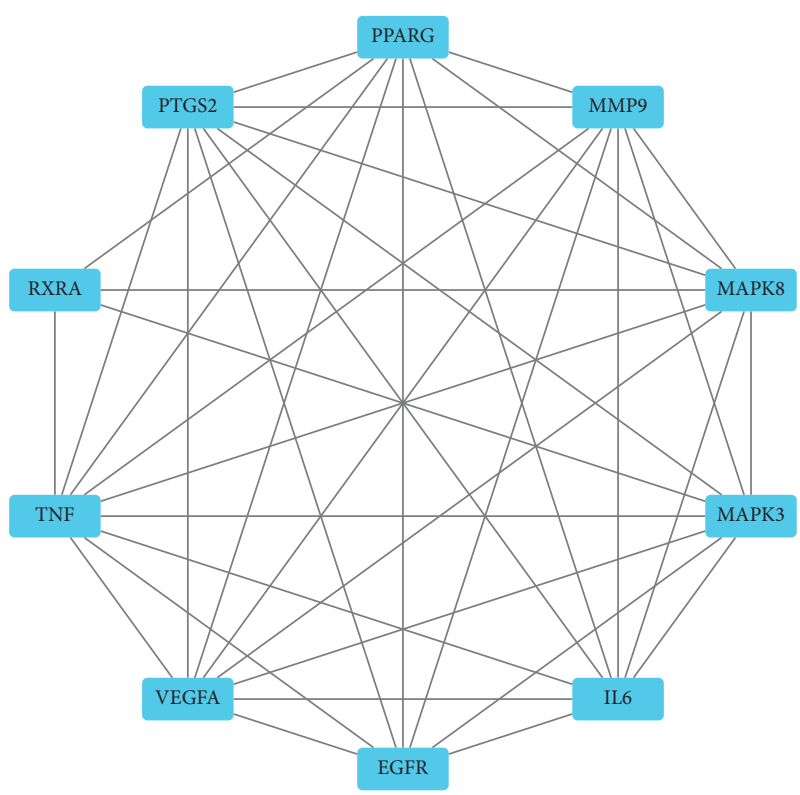

(b)

Figure 3: PPI network of targets for FGHP against CHD (a). Top 10 targets in PPI network (b).

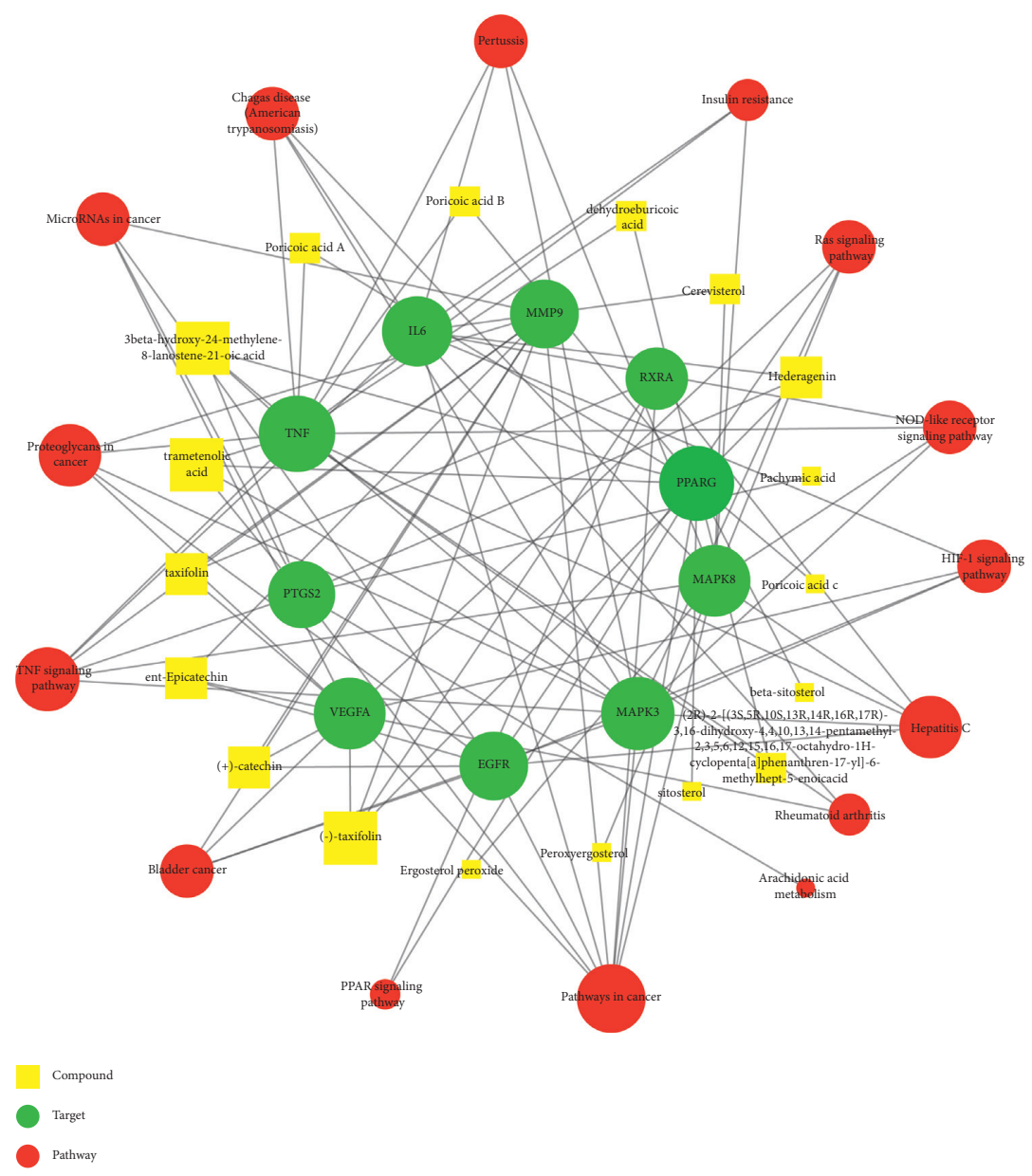

Figure 4: The component-target-pathway network. There is a positive proportional relationship between the node size and the edge count. 

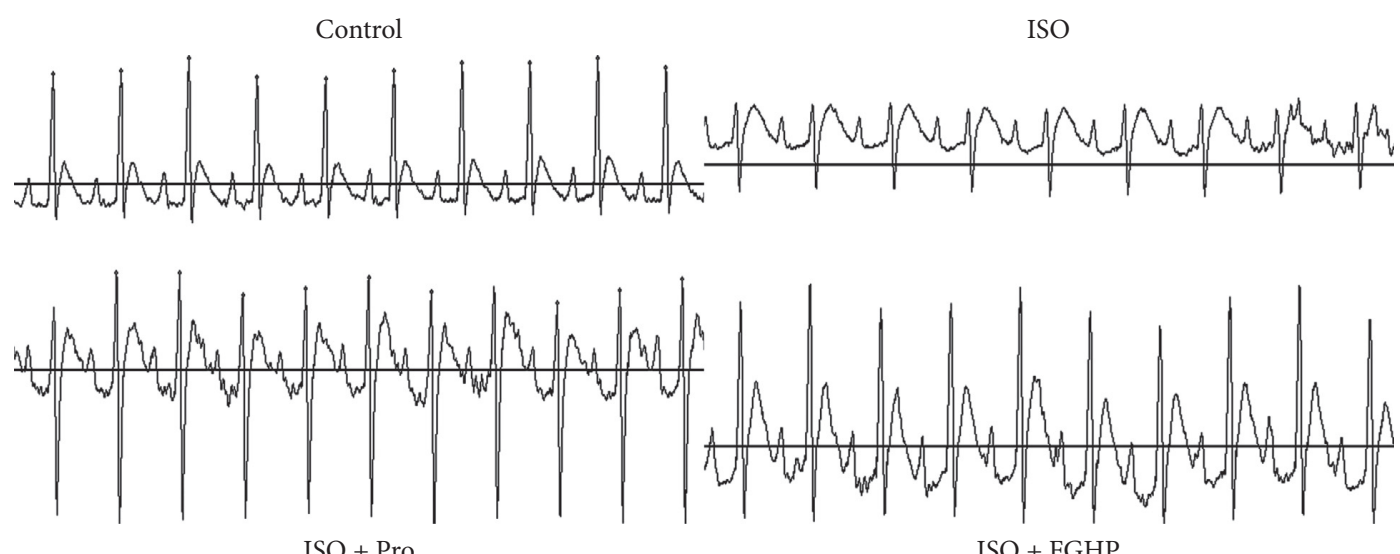

(a)

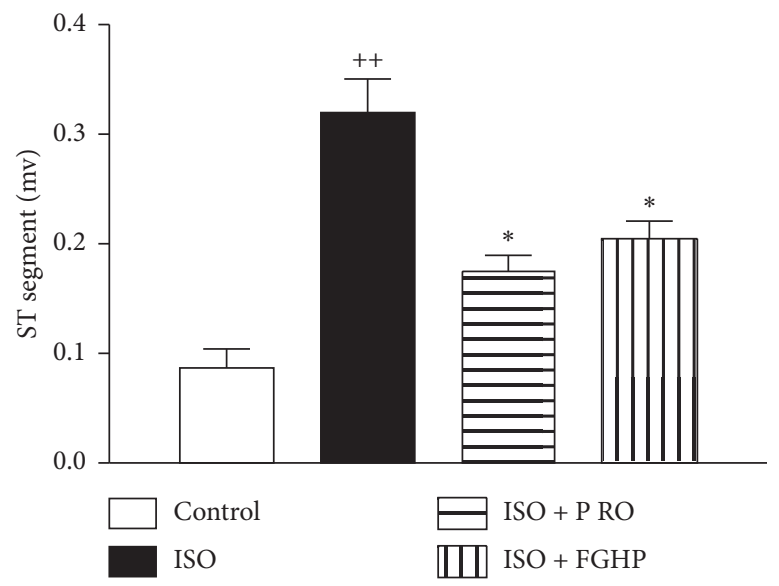

(b)

FIGURE 5: Effects of FGHP on ECG in AMI rats. ECG recordings (a) and bar graph of ST segment (b). ${ }^{++} P<0.01$ vs. the control group; ${ }^{*} P<0.05$ vs. the ISO group. Values are presented as mean \pm SEM.

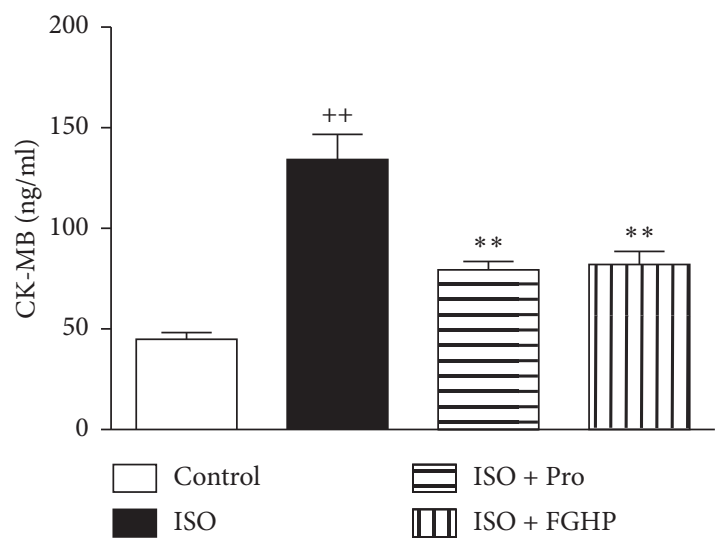

(a)

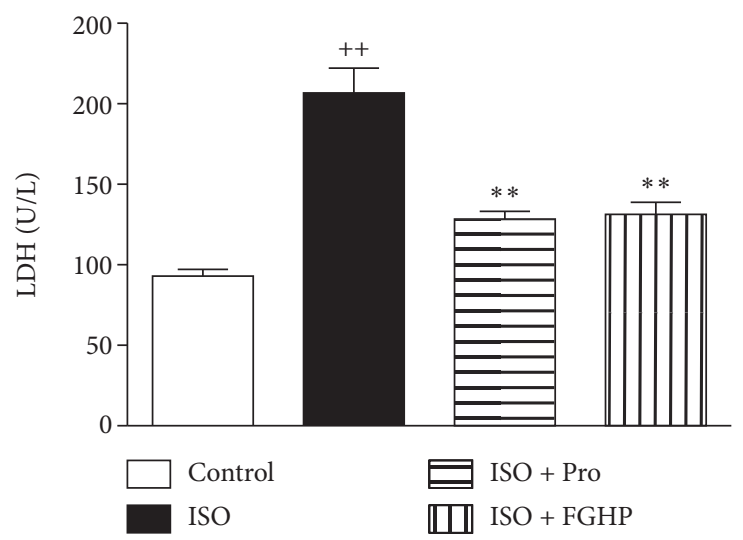

(b)

FIGURE 6: Effects of FGHP on CK-MB (a) and LDH (b) in AMI rats. ${ }^{++} P<0.01$ vs. the control group; ${ }^{* *} P<0.01$ vs. the ISO group. Values are presented as mean \pm SEM.

In in vivo studies, we attempted to study the therapeutic effect of FGHP and confirm the predicted results from network pharmacology. To this end, we tested this agent in rats with ISO-induced AMI. Our results showed that characteristic indices including CK-MB and LDH content were decreased significantly in FGHP pretreated rats 


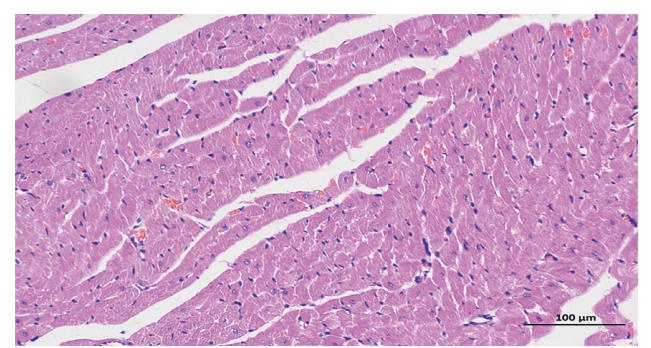

(a)

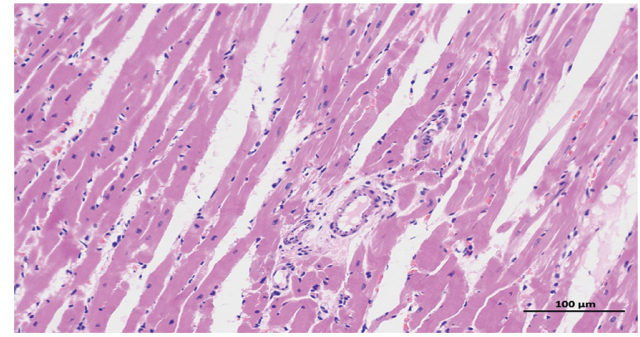

(c)

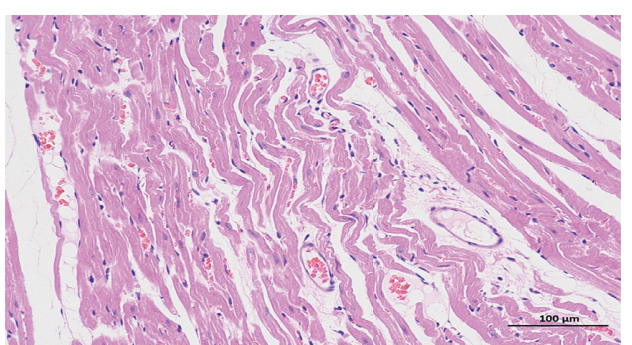

(b)

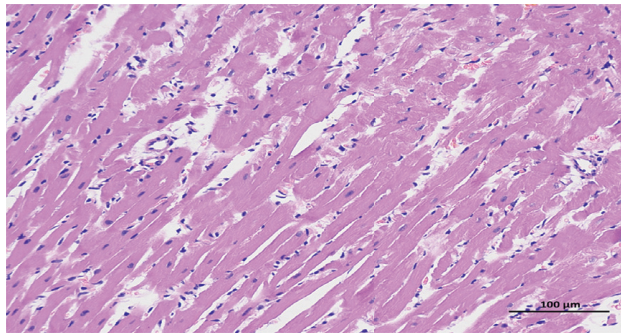

(d)

FIGURE 7: Effects of FGHP on histopathological features in AMI rats. Representative H\&E staining images of heart paraffin sections in the various groups. Original magnification $\times 200$. (a) Control. (b) ISO. (c) ISO + Pro. (d) ISO + FGHP.
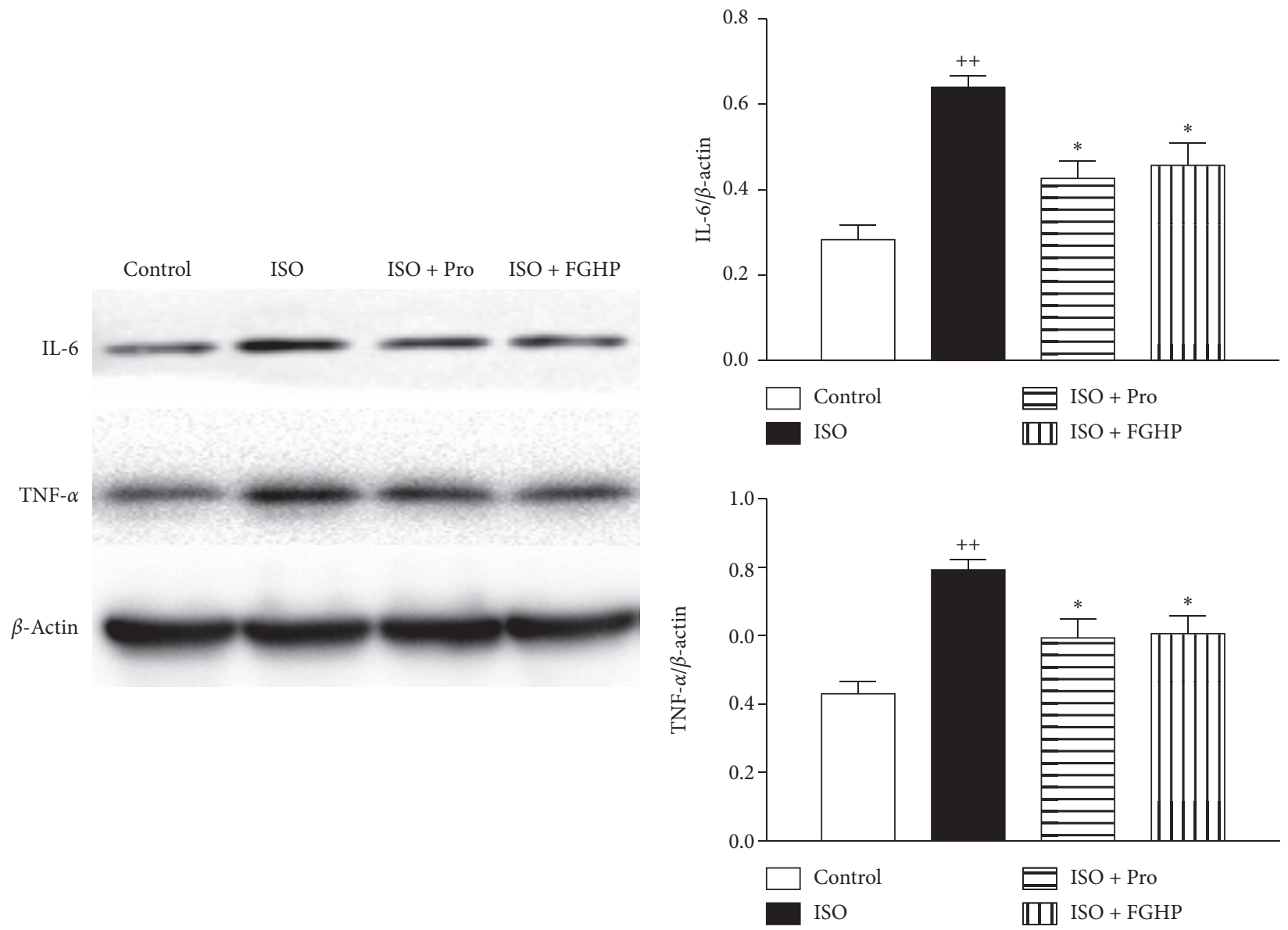

FIGURE 8: Effects of FGHP on TNF- $\alpha$ and IL-6 in cardiac muscle in AMI rats by western blot analysis. ${ }^{++} P<0.01$ vs. the control group; ${ }^{*} P<0.05$ vs. the ISO group. Values are presented as mean \pm SEM. 
compared with AMI control rats (shown in Figure 6). In addition, FGHP could ameliorate the ECG (shown in Figure 5) and myocardial tissue abnormalities in ISO-induced AMI rats to some extent (shown in Figure 7). These results indicated that FGHP could impart a therapeutic effect on acute myocardial ischemia.

Moreover, we further verify the pharmacological mechanism of FGHP on treatment for CHD by measuring the expression levels of the core and specific targets. Upregulated TNF- $\alpha$ and IL- 6 expressions were detected in myocardial tissues in the ISO group rats; however, both of them were significantly reduced with FGHP pretreatment (shown in Figure 8). These findings were accompanied by decreased inflammatory cell invasion and improved pathological changes (Figure 7), indicating that suppression of TNF- $\alpha$ and IL- 6 activity to modulate inflammation is at least in part responsible for FGHP exerting its cardiac protective efficacy. Further molecular mechanism studies and confirmation of the prediction of FGHP are required.

\section{Conclusion}

In summary, our study predicted and verified that FGHP could reduce protein expression of TNF- $\alpha$ and IL- 6 for the suppression of inflammatory response in cardiac muscle to exert curative effect for CHD. The results partially explained the pharmacological mechanism of FGHP against CHD.

\section{Data Availability}

The data used to support the findings of this study are available from the corresponding author upon request.

\section{Conflicts of Interest}

The authors declare that there are no conflicts of interest regarding the publication of this paper.

\section{Authors' Contributions}

Fang Huang and Guangyu Tian conceived and designed the study. Shuping Ming and Lintao Han analyzed the data. Bailu Duan wrote the manuscript. Bailu Duan, JingJing Li, Qiong Wang, and Dongning Zhang carried out the experiments. All authors read and approved the final manuscript.

\section{Acknowledgments}

The project was supported by the Beanstalk Program of Hubei University of Chinese Medicine (2017ZZX011).

\section{Supplementary Materials}

Supplementary Table S1: the detailed information of ingredients in FL and GZ. Supplementary Table S2: the detailed target information of compounds in FGHP. Supplementary Table S3: targets related to CHD. Supplementary Table S4: overlapping targets between FGHP and CHD. Supplementary Table S5: GO and pathway enrichment analysis by DAVID. (Supplementary Materials)

\section{References}

[1] X. Xu, H. Hu, Y. Lin et al., "Differences in leukocyte telomere length between coronary heart disease and normal population: a multipopulation meta-analysis," BioMed Research International, vol. 2019, Article ID 5046867, 9 pages, 2019.

[2] X. Yang, T. He, S. Han et al., "The role of traditional Chinese medicine in the regulation of oxidative stress in treating coronary heart disease," Oxidative Medicine and Cellular Longevity, vol. 2019, Article ID 3231424, 13 pages, 2019.

[3] https://www.who.int/health-topics/cardiovascular-diseases.

[4] R. Chen, Ya Xiao, M. Chen et al., "A traditional Chinese medicine therapy for coronary heart disease after percutaneous coronary intervention: a meta-analysis of randomized, double-blind, placebo-controlled trials," Bioscience Reports, vol. 38, no. 5, Article ID 30143584, 2018.

[5] M. Orho-Melander, "Genetics of coronary heart disease: towards causal mechanisms, novel drug targets and more personalized prevention," Journal of Internal Medicine, vol. 278, no. 5, pp. 433-446, 2015.

[6] Y. Zhao, S. Nie, M. Yi et al., "UPLC-QTOF/MS-based metabolomics analysis of plasma reveals an effect of Xue-Fu$\mathrm{Zhu}-\mathrm{Yu}$ capsules on blood-stasis syndrome in CHD rats," Journal of Ethnopharmacology, vol. 241, Article ID 111908, 2019.

[7] M. Ding, W. Ma, X. Wang et al., "A network pharmacology integrated pharmacokinetics strategy for uncovering pharmacological mechanism of compounds absorbed into the blood of Dan-Lou tablet on coronary heart disease," Journal of Ethnopharmacology, vol. 242, Article ID 112055, 2019.

[8] C. Wang, M. Niimi, T. Watanabe, Y. Wang, J. Liang, and J. Fan, "Treatment of atherosclerosis by traditional Chinese medicine: questions and quandaries," Atherosclerosis, vol. 277, pp. 136-144, 2018.

[9] D. Yan, X. R. Xu, B. W. Yue, L. L. Zhao, S. H. Yan, and Y. Qian, "How to continue lipid- lowering therapy in patients with coronary heart disease and severe liver dysfunction ?: a case report of plaque regression by a combination of Chinese and western medicine," Medicine (Baltimore), vol. 98, no. 43, Article ID e17664, 2019.

[10] S. Wang, Y. Hu, W. Tan et al., "Compatibility art of traditional Chinese medicine: from the perspective of herb pairs," Journal of Ethnopharmacology, vol. 143, no. 2, pp. 412-423, 2012.

[11] B. Boezio, K. Audouze, P. Ducrot, and O. Taboureau, "Network-based approaches in pharmacology," Molecular Informatics, vol. 36, no. 10, Article ID 28692140, 2017.

[12] H. Yuan, Ma, Cui, Z. Liu et al., "How can synergism of traditional medicines benefit from network pharmacology?" Molecules, vol. 22, no. 7, Article ID E1135, 2017.

[13] R. Zhang, X. Zhu, H. Bai, and K. Ning, "Network pharmacology databases for traditional Chinese medicine: review and assessment," Frontiers in Pharmacology, vol. 10, p. 123, 2019.

[14] D. Szklarczyk, J. H. Morris, H. Cook et al., "The STRING database in 2017: quality-controlled protein-protein association networks, made broadly accessible," Nucleic Acids Research, vol. 45, no. D1, pp. D362-D368, 2017.

[15] B. Demchak, T. Hull, M. Reich et al., "Cytoscape: the network visualization tool for genome space workflows," F1000Research, vol. 3, p. 151, 2014.

[16] Q. Yang, D. D. Huang, D. G. Li et al., "Tetramethylpyrazine exerts a protective effect against injury from acute myocardial ischemia by regulating the PI3K/Akt/GSK-3 $\beta$ signaling pathway," Cellular \& Molecular Biology Letters, vol. 24, no. 1, p. 17, 2019. 
[17] J. Lu, Y. Hu, L. Wang et al., "Understanding the multitarget pharmacological mechanism of the traditional Mongolian common herb pair GuangZao-RouDouKou acting on coronary heart disease based on a bioinformatics approach," Evidence-Based Complementary and Alternative Medicine, vol. 2018, Article ID 7956503, 12 pages, 2018.

[18] H. Xu, J. Zou, X. Ye et al., "Impacts of clinical pharmacist intervention on the secondary prevention of coronary heart disease: a randomized controlled clinical study," Frontiers in Pharmacology, vol. 10, p. 1112, 2019.

[19] Y. Yang, C. Huang, X. Su et al., "Deciphering the multicomponent synergy mechanism from a systems pharmacology perspective: application to Gualou Xiebai decoction for coronary heart disease," Journal of Functional Foods, vol. 47, pp. 143-155, 2018.

[20] Z. Yan, Z. Lai, and J. Lin, “Anticancer properties of traditional Chinese medicine," Combinatorial Chemistry \& High Throughput Screening, vol. 20, no. 5, pp. 423-429, 2017.

[21] Z. Lu, Y. Zhong, W. Liu, L. Xiang, and Y. Deng, “The efficacy and mechanism of Chinese herbal medicine on diabetic kidney disease," Journal of Diabetes Research, vol. 2019, Article ID 2697672, 14 pages, 2019.

[22] R. Z. Zhang, S. J. Yu, H. Bai, and K. Ning, “TCM-mesh: the database and analytical system for network pharmacology analysis for TCM preparations," Scientific Reports, vol. 7, no. 1, p. 2821, 2017.

[23] J. Zhang, R. Liang, L. Wang, and B. Yang, "Effects and mechanisms of Danshen-Shanzha herb-pair for atherosclerosis treatment using network pharmacology and experimental pharmacology," Journal of Ethnopharmacology, vol. 229, pp. 104-114, 2019.

[24] B. Liu, Z. Song, J. Yu, P. Li, Y. Tang, and J. Ge, "The atherosclerosis-ameliorating effects and molecular mechanisms of BuYangHuanWu decoction," Biomed Pharmacother, vol. 123, Article ID 109664, 2020.

[25] W. Wang, T. Liu, L. Yang et al., "Study on the multi-targets mechanism of triphala on cardio-cerebral vascular diseases based on network pharmacology," Biomedicine \& Pharmacotherapy, vol. 116, Article ID 108994, 2019.

[26] H. Li, K. Sun, R. Zhao et al., "Inflammatory biomarkers of coronary heart disease," Frontiers in Bioscience, vol. 10, no. 1, pp. 185-196, 2018.

[27] H. Teague and N. N. Mehta, "The link between inflammatory disorders and coronary heart disease: a look at recent studies and novel drugs in development," Current Atherosclerosis Reports, vol. 18, no. 1, p. 3, 2016.

[28] Y. Xu, H. Hu, Y. Li et al., "Effects of huoxin formula on the arterial functions of patients with coronary heart disease," Pharmaceutical Biology, vol. 57, no. 1, pp. 13-20, 2019.

[29] M. Cainzos-Achirica, C. Enjuanes, P. Greenland et al., "The prognostic value of interleukin 6 in multiple chronic diseases and all-cause death: the multi-ethnic study of atherosclerosis (MESA)," Atherosclerosis, vol. 278, pp. 217-225, 2018.

[30] Y. Dong, H. Chen, J. Gao, Y. Liu, J. Li, and J. Wang, "Molecular machinery and interplay of apoptosis and autophagy in coronary heart disease," Journal of Molecular and Cellular Cardiology, vol. 136, pp. 27-41, 2019.

[31] K. Pulido-Gómez, Y. Hernández-Díaz, C. A. Tovilla-Zárate et al., "Association of G308A and G238A polymorphisms of the TNF- $\alpha$ gene with risk of coronary heart disease: systematic review and meta-analysis," Archives of Medical Research, vol. 47, no. 7, pp. 557-572, 2016.

[32] J. Yuepeng, X. Zhao, Y. Zhao, and L. Li, "Gene polymorphism associated with TNF- $\alpha$ (G308A) IL-6 (C174G) and susceptibility to coronary atherosclerotic heart disease: a meta-analysis," Medicine (Baltimore), vol. 98, no. 2, Article ID e13813, 2019.

[33] C. H. Junmei and Z. H. Chunxiang, "Advances in pharmacological mechanism of Linggui zhugan tang," Chinese Journal of Experimental Traditional Medical Formulae, vol. 25, no. 14, pp. 222-227, 2019, in Chinese.

[34] S. Hui, W. Liang, H. Jinling et al., "Effect of Linggui zhugan decoction containing serum on protein expression of IKK/ $\mathrm{I} \kappa \mathrm{B} / \mathrm{NF}-\kappa \mathrm{B}$ signal pathways in lipopolysaccharide-induced cardiomyocyte injury model rats," Chinese Journal of Integrated Traditional and Western Medicine, vol. 37, no. 10, pp. 1215-1219, 2017, in Chinese.

[35] L. Qiongqiong, H. Jun, Z. Baihui et al., "Connotation research on "treating same disease with different methods" of Linggui Zhugan decoction and shenqi pill based on CHF rat model," China Journal of Traditional Chinese Medicine and Pharmacy, vol. 34, no. 2, pp. 537-576, 2019, in Chinese.

[36] G. J. Kim, D. H. Song, H. S. Yoo, K. H. Chung, K. J. Lee, and J. H. An, "Hederagenin supplementation alleviates the proinflammatory and apoptotic response to alcohol in rats," Nutrients, vol. 9, no. 1, p. 41, 2017.

[37] Y. Yin, X. Liu, J. Liu et al., "Beta-sitosterol and its derivatives repress lipopolysaccharide/d-galactosamine-induced acute hepatic injury by inhibiting the oxidation and inflammation in mice," Bioorganic \& Medicinal Chemistry Letters, vol. 28, no. 9, pp. 1525-1533, 2018. 